\title{
Use of Doehlert Designs for Second-order Polynomial Models
}

\author{
L. Rob Verdooren \\ Danone Nutricia Research, Netherlands
}

Copyright $\bigcirc 2017$ by authors, all rights reserved. Authors agree that this article remains permanently open access under the terms of the Creative Commons Attribution License 4.0 International License

\begin{abstract}
The most popular designs for fitting the second-order polynomial model are the central composite designs of Box and Wilson [2] and the designs of Box and Behnken [1]. For $k=2,4,6$ and 8, the uniform shell designs of Doehlert [4] require fewer experimental runs than the central composite or Box-Behnken designs. In analytic chemistry the Doehlert designs are widely used. The uniform shell designs are based on a regular simplex, this is the geometric figure formed by $k+1$ equally spaced points in a $k$ - dimensional space; an equilateral triangle is a two-dimensional regular simplex. The shell designs are used for fitting a response surface to $k$ independent factors over a spherical region. Doehlert $(1930$ - 1999) proposed in 1970 the design for $k=2$ factors starting from an equilateral triangle with sides of length 1 , to construct a regular hexagon with a centre point at $(0,0)$. The $n=7$ experimental points are $(1,0),(0.5,0.866),(0,0),(-0.5,0.866),(-1,0),(-0.5$, $-0.866)$ and $(0.5,-0.866)$. The 6 outer points lie on a circle with a radius 1 and centre $(0,0)$. This Doehlert design has an equally spaced distribution of points over the experimental region, a so-called uniform space filler, where the distances between neighboring experiments are equal. Response surface designs are usually applied by scaling the coded factor ranges to the ranges of the experimental factors. The first factor covers the interval $[-1,+1]$, the second factor covers the interval $[-0.866,+0.866]$. Doehlert design for four factors needs only 21 trials. Doehlert and Klee [5] show how to rotate the uniform shell designs to minimize the number of levels of the factors. Most of the rotated uniform shell designs have no more than five levels of any factor; the central composite design has five levels of every factor. The D-Optimality determinant criterion of the variance matrix of Doehlert designs will be compared with central composite designs and Box-Behnken designs, see Rasch et al. [6].
\end{abstract}

Keywords Second-order Polynomial Designs, Quadratic Response Designs, Doehlert Designs

\section{Introduction}

The most popular designs for fitting the second-order polynomial model are the central composite designs of Box and Wilson [2] and the designs of Box and Behnken [1].

For $k=2,4,6$ and 8, the uniform shell designs of Doehlert [4] require fewer experimental runs than the central composite or Box-Behnken designs. In analytic chemistry the Doehlert designs are widely used. The uniform shell designs are based on a regular simplex, this is the geometric figure formed by $k+1$ equally spaced points in a $k-$ dimensional space; an equilateral triangle is a two-dimensional regular simplex. The shell designs are used for fitting a response surface to $k$ independent factors over a spherical region.

The second-order polynomial model for $k=2$ factors and $n$ experimental units is:

$$
\begin{aligned}
& \mathrm{E}\left(\boldsymbol{y}_{i}\right)=\beta_{0}+\beta_{1} \mathrm{X}_{1 i}+\beta_{2} \mathrm{X}_{2 i}+\beta_{11} \mathrm{X}_{1 i}^{2}+ \\
& +\beta_{22} \mathrm{X}_{2 i}^{2}+\beta_{12} \mathrm{X}_{1 i} \mathrm{X}_{2 i} \quad(i=1,2, \ldots, n) .
\end{aligned}
$$

There are npar $=6$ parameters and to obtain the least squares estimators for the parameters, the factors $X_{1}$ and $X_{2}$ must have at least three different levels for the second-order polynomial and the number of experimental units $n$ must be larger than 6, the number of parameters npar.

Doehlert (1930 - 1999) proposed in 1970 the design for $k$ $=2$ factors $X_{1}$ and $X_{2}$, starting from an equilateral triangle with sides of length 1 , to construct a regular hexagon with a centre point at $(0,0)$. 


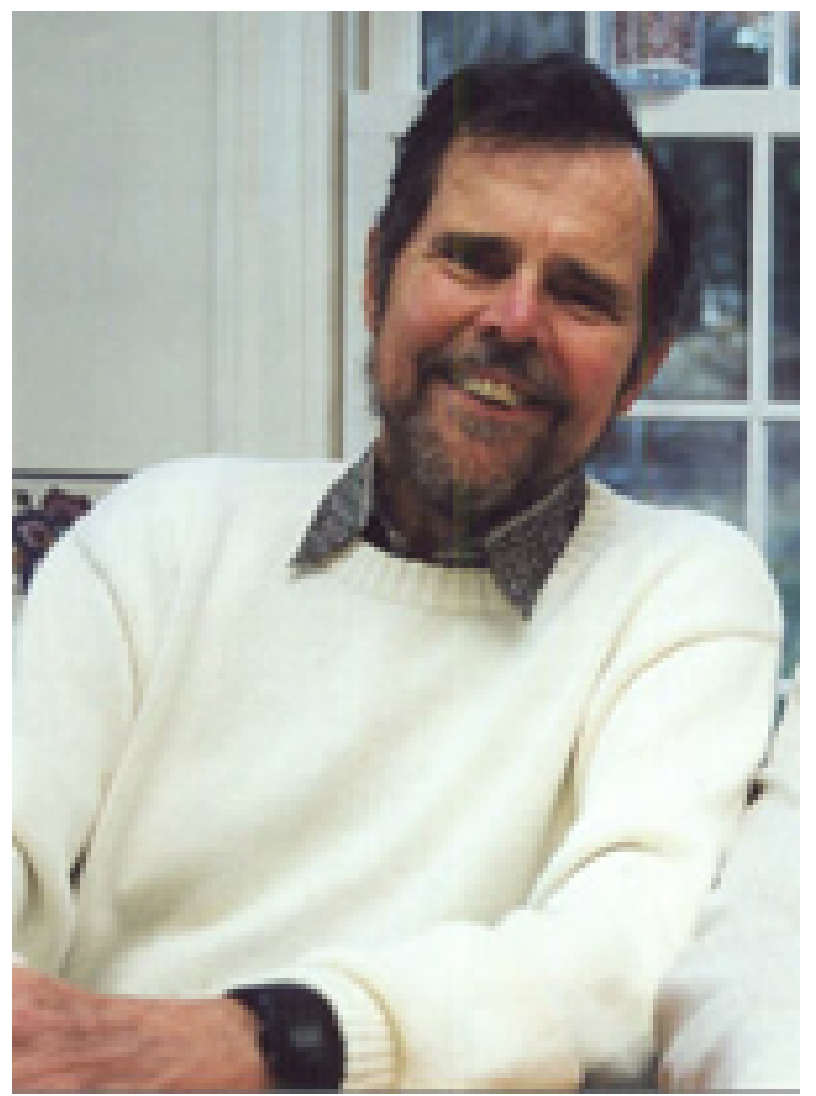

David H. Doehlert (1930 - 1999)

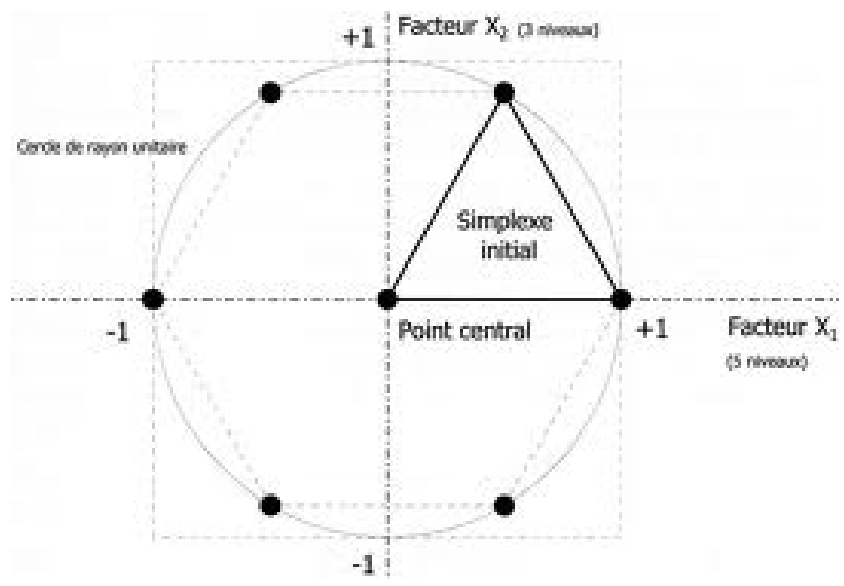

The $n=7$ experimental units (points) of the Doehlert design with $k=2$ factors.

$\begin{array}{llll}\mathrm{X}_{1} & \mathrm{X}_{2} & \mathrm{X}_{1} & \mathrm{X}_{2} \\ \cos (0) & \sin (0) & 1 & 0 \\ \cos (\pi / 3) & \sin (\pi / 3) & 0.5 & 0.866 \\ \cos (2 \pi / 3) & \sin (2 \pi / 3) & -0.5 & 0.866 \\ \cos (\pi) & \sin (\pi) & -1 & 0 \\ \cos (4 \pi / 3) & \sin (4 \pi / 3) & -0.5 & -0.866 \\ \cos (5 \pi / 3) & \sin (5 \pi / 3) & 0.5 & -0.866 \\ 0 & 0 & 0 & 0\end{array}$

The 6 outer points lie on a circle with a radius 1 and the inner point is the centre $(0,0)$. This Doehlert design has an equally spaced distribution of points over the experimental region, a so-called uniform space filler, where the distances between neighboring experimental units are equal. Response surface designs are usually applied by scaling the coded factor ranges to the ranges of the experimental factors. The first factor covers the interval $[-1,+1]$, the second factor covers the interval $[-0.866,+0.866]$.

If the desired results are not found in the first study domain (A), the domain can be extended in the direction where the most of the desired points are likely to be. Just add three experimental units to find a new Doehlert design (B) which forms again a regular hexagon, and further add again three experimental units to find another new Doehlert design (C).

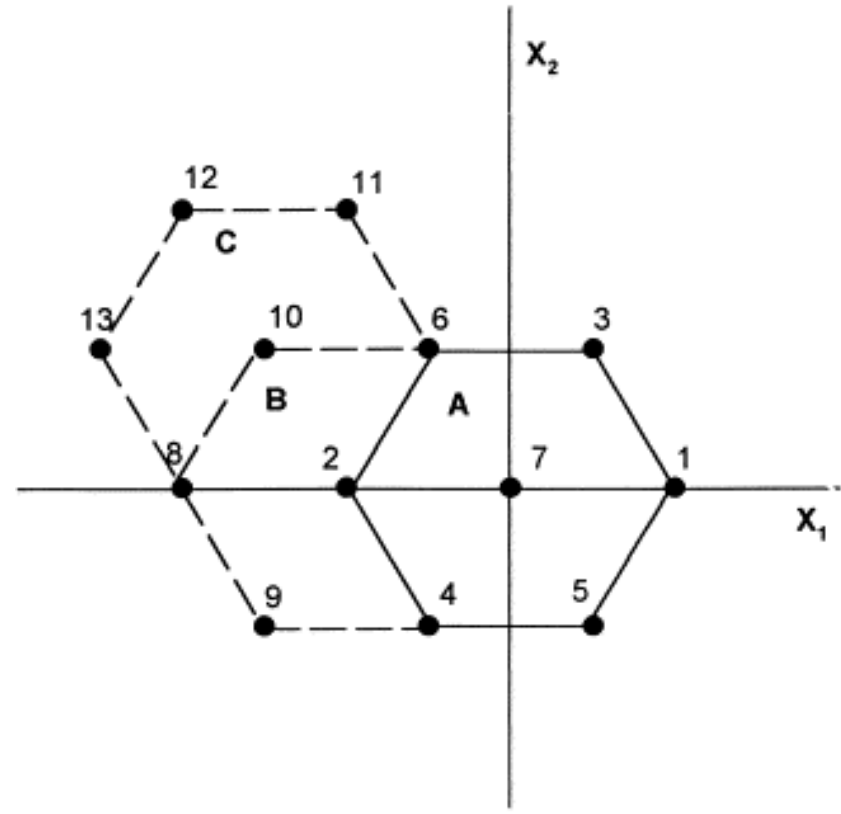

\begin{tabular}{llll}
\hline $\begin{array}{l}\text { Experimental } \\
\text { matrix }\end{array}$ & Exp. & $\mathrm{X}_{1}$ & $\mathrm{X}_{2}$ \\
\hline $\mathrm{A}$ & $\mathrm{1}$ & 1 & 0 \\
& 2 & -1 & 0 \\
& 3 & 0.5 & 0.866 \\
& 4 & -0.5 & -0.866 \\
& 5 & 0.5 & -0.866 \\
& 6 & -0.5 & 0.866 \\
& 7 & 0 & 0 \\
\hline $\mathrm{B}$ & 8 & -2 & 0 \\
& 9 & -1.5 & -0.866 \\
& 10 & -1.5 & 0.866 \\
\hline $\mathrm{C}$ & 11 & -1 & 1.732 \\
& 12 & -2 & 1.732 \\
& 13 & -2.5 & 0.866 \\
\hline
\end{tabular}

All the points in the Doehlert design for $k=2$ factors are on a unit circle (in centered and scaled units). The domain defined by Doehlert designs is spherical: a circle in two dimensions, a sphere in three dimensions for $k=3$ factors, a hypersphere in more than three dimensions for $k>3$ factors. 


\section{Doehlert Designs for 2, 3, 4 and 5 Factors}

Below are given the Doehlert designs for $k=2$ factors $X_{1}$ and $X_{2}$, with experimental unit (trial) 1-7; for $k=3$ factors $X_{1}, X_{2}$ and $\mathrm{X}_{3}$, trial 1-13; for $k=4$ factors $\mathrm{X}_{1}, \mathrm{X}_{2}, \mathrm{X}_{3}$ and $\mathrm{X}_{4}$, trial 1-21; for $k=5$ factors $\mathrm{X}_{1}, \mathrm{X}_{2}, \mathrm{X}_{3}, \mathrm{X}_{4}$ and $\mathrm{X}_{5}$, trial 1-31.

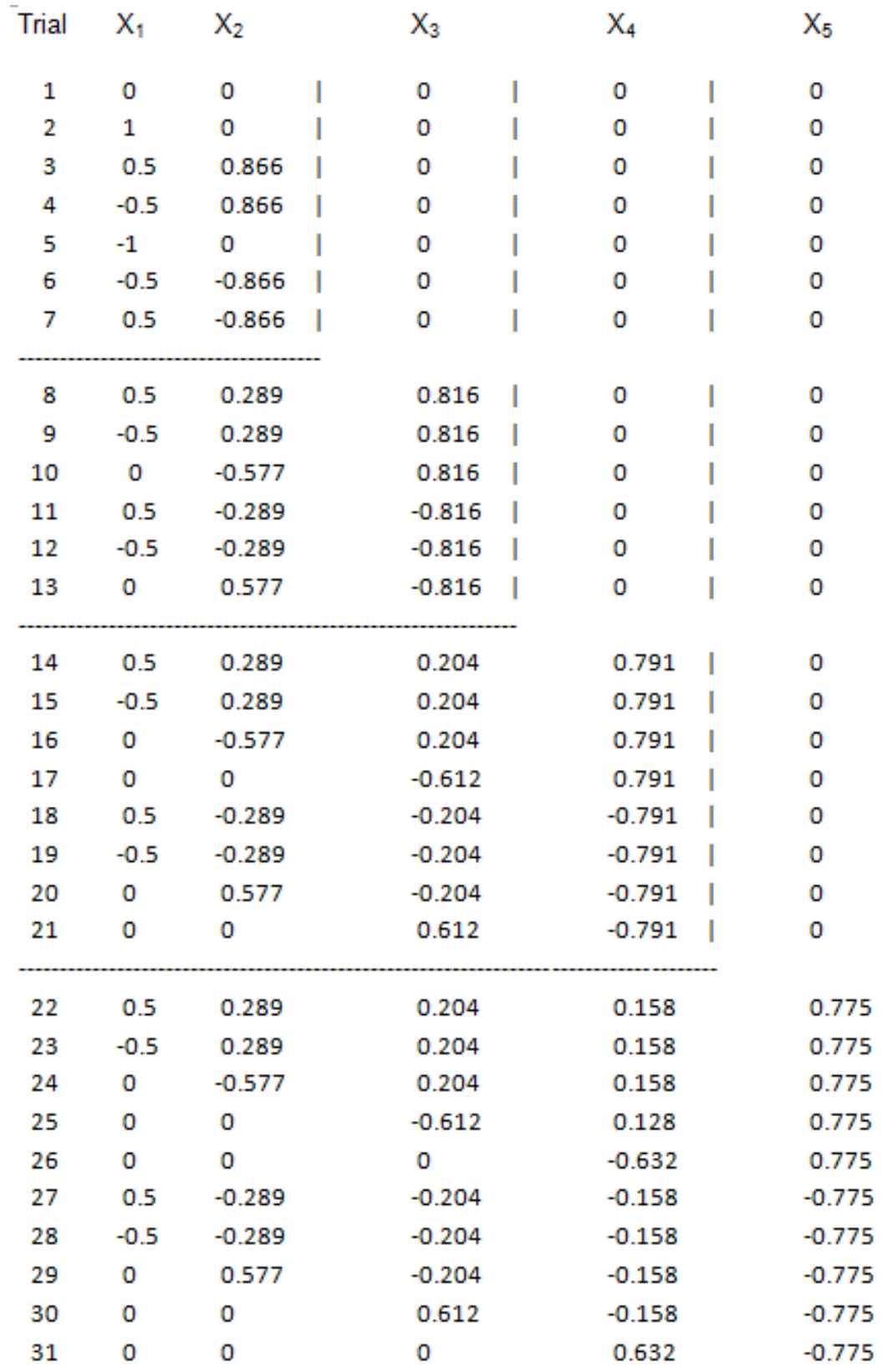

\section{Rotation of Doehlert Designs}

The Doehlert designs can be rotated to change the design region. Furthermore the symmetry of the design matrix can be changed by a rotation of the design.

The rotation will be demonstrated for $k=2$ factors $\mathrm{X}_{1}$ and $\mathrm{X}_{2}$ with a regular hexagon design.

We rotate over an angle of $\pi / 12$ clockwise. This means that the coordinates $\left(\mathrm{X}_{1}, \mathrm{X}_{2}\right)$ are multiplied with the matrix

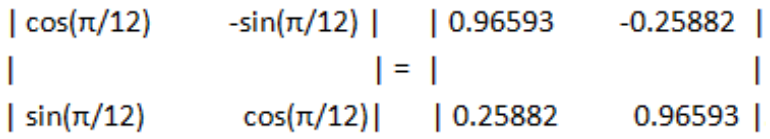


Afterwards we want that the range of the new coordinates is $[-1,+1]$, hence we must multiply the rotated coordinates with $1 / 0.96593=1.035272$.

\begin{tabular}{ccccccl}
\multicolumn{4}{c}{$\begin{array}{l}\text { Original } \\
\text { coordinates }\end{array}$} & \multicolumn{2}{l}{\begin{tabular}{l}
\multicolumn{2}{l}{ Rotation over } \\
Point coordinates
\end{tabular}} \\
& $\mathrm{X}_{1}$ & $\mathrm{X}_{2}$ & $\mathrm{X}_{1}{ }^{\prime}$ & $\mathrm{X}_{2}{ }^{\prime}$ & $\mathrm{X}_{1}{ }^{*}$ & $\mathrm{X}_{2}{ }^{*}$ \\
1 & 1 & 0 & 0.966 & -0.259 & 1 & -0.268 \\
2 & 0.5 & -0.866 & 0.259 & -0.966 & 0.268 & -1 \\
3 & -0.5 & -0.866 & -0.707 & -0.707 & -0.732 & -0.732 \\
4 & -1 & 0 & -0.966 & 0.259 & -1 & 0.268 \\
5 & -0.5 & 0.866 & -0.259 & 0.966 & -0.268 & 1 \\
6 & 0.5 & 0.866 & 0.707 & 0.707 & 0.732 & 0.732 \\
7 & 0 & 0 & 0 & 0 & 0 & 0
\end{tabular}

Before rotation the factor $\mathrm{X}_{1}$ has range $[-1,1]$ and the factor $\mathrm{X}_{2}$ has range $[-0.866,0.866]$.

After rotation and scaling the two factors have the range $[-1,+1]$.

Before the rotation factor $\mathrm{X}_{1}$ has 5 levels $(-1,-0.5,0,0.5,1)$ and factor $\mathrm{X}_{2}$ has 3 levels $(-0.866,0,0,866)$. After rotation and scaling the two factors $\mathrm{X}_{1}{ }^{*}$ and $\mathrm{X}_{2}{ }^{*}$ have 7 levels $(-1,-0.732,-0.268,0,0.268,0.732,1)$.

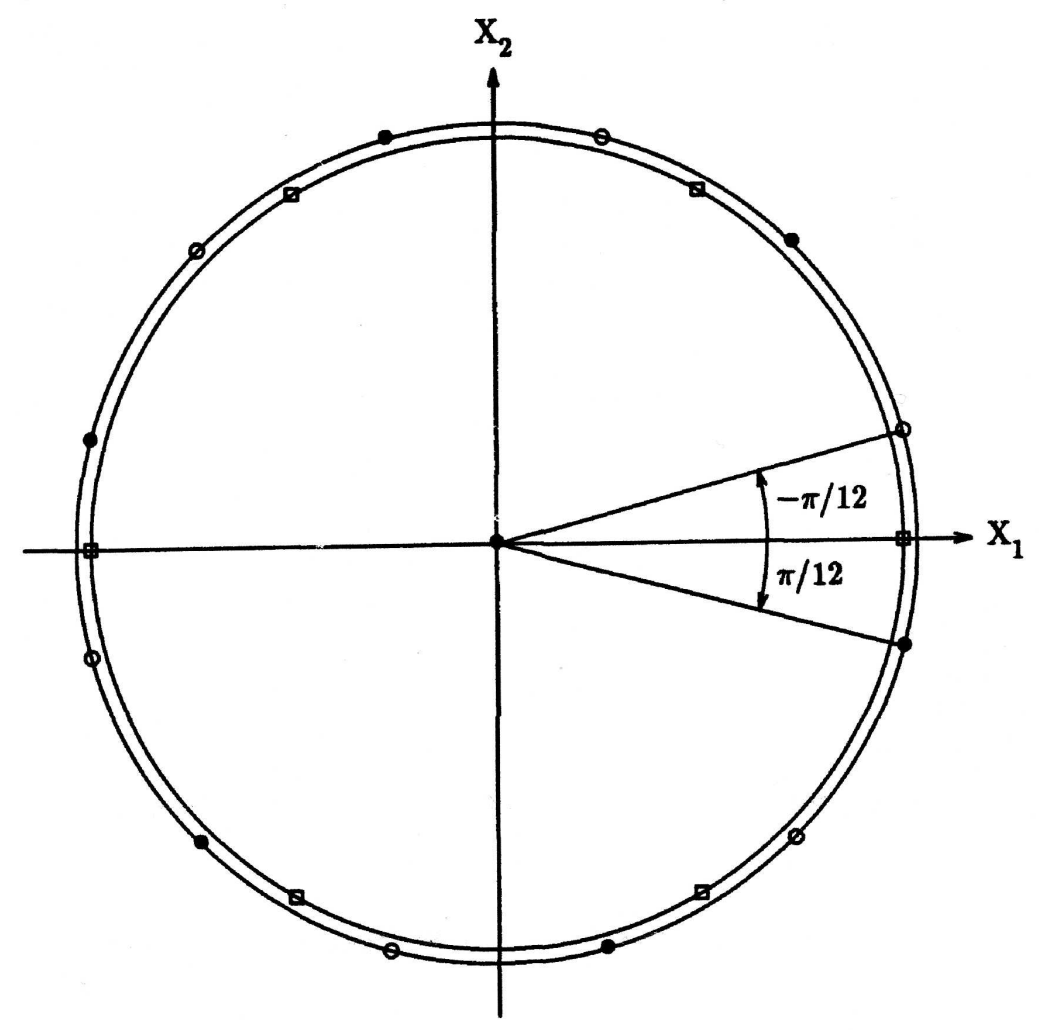

Two Rotations and Scalings of the Hexagon Design. The hexagon design in ice usual orientation is indicated by small squares. After rotation by $\pi / 12$ radians and scaling the factor levels to the range -1 to 1 , the design points are indicated by a solid dots for a clockwise ratation of the design and by hollow dots for a counterclockwise rotation of the design. Notice that the clockwise and counterclockwise rotations are reflections across the $x_{1}$-axis; multiplying the second coordinate by -1 will change one rotation into the other. 
Doehlert and Klee [5] show how to rotate the uniform shell designs to minimize the number of levels of the factors. Most of the rotated uniform shell designs have no more than five levels of any factor. Note that the central composite design has five levels of every factor.

\section{Comparison of Number of Runs for Second Order Polynomial Designs}

In the following table the number of factors $k=2,3,4$ and 5 is given with the number of parameters npar of the second-order polynomial, the minimum number $n$ of the experimental units for the Doehlert design, the Central Composite Design (CCD) when it is rotatable and non-orthogonal (CCD1) and for the Central Composite Design when it is rotatable and orthogonal (CCD2). (If the variance of the estimator of $\mathrm{E}(\mathrm{y})$ at all points from the centre point is constant then the design is called rotatable. If the columns of the design matrix $\mathrm{X}$ in $\mathrm{E}(\mathrm{y})=\mathrm{X} \beta$ are orthogonal, the design is called orthogonal.) Further for $k=3,4$ and 5 the minimal $n$ is given for the Box-Behnken (BB) designs.

$\begin{array}{llcccl} & & \text { Doehlert } & \text { CCD1 } & \text { CCD2 } & \text { BB } \\ k & n \text { npar } & n & n & n & n \\ 2 & 6 & 7 & 9 & 16 & \\ 3 & 10 & 13 & 15 & 32 & 13 \\ 4 & 15 & 21 & 25 & 36 & 25 \\ 5 & 21 & 31 & 27 & 36 & 41\end{array}$

\section{D-Optimality Criterion Comparison for Designs with 2 or 3 Factors}

To investigate optimality criterion of the design, the determinant criterion of the variance matrix of Doehlert designs will be compared with central composite designs and Box-Behnken designs, see Rasch et al. [6]. The criterion of the $D$-optimality requires the minimization of the determinant of the variance-covariance matrix of the estimator of the vector of regression coefficient, which equals $\sigma^{2}\left(X^{\mathrm{T}} \mathrm{X}\right)^{-1}$. We put without loss of generality $\sigma^{2}=1$ and use besides critI $=\operatorname{det}\left[\left(\mathrm{X}^{\mathrm{T}} \mathrm{X}\right)^{-1}\right]$ which has to be minimized, a modified criterion critII $=\left\{\operatorname{det}\left[\left(\mathrm{X}^{\mathrm{T}} \mathrm{X}\right)^{-1}\right]\right\}^{1 / k}$ which has to be maximized. The resulting design is of course the same in both cases; the second criterion is easier to handle. We use however a criterion proposed by Draper and Lin [3]: critIII $=c r i t I I / n=\left\{\operatorname{det}\left[\left(\mathrm{X}^{\mathrm{T}} \mathrm{X}\right)^{-1}\right]\right\}^{1 / k} / n$.

When we compare for $k=2$ factors the CCD1 $(\mathrm{n}=9)$ with a Doehlert design, where we add two extra centre points $(0,0)$ to get also $n=9$ experimental units, we have for this Doehlert design with $n=9$ critI $=1 / 91.10362=0.010977$ and critIII $=$ 1.060536. This Doehlert design is rotatable.

The CCD1 with the star points at -1 and +1 , is called an "inscribed Central Composite Design" because the circle with the experimental units has the radius 1 ; the distance of the star points to the centre $(0,0)$. This $C C D 1$ has the 4 cube points $(-0.7071,-0.7071),(-0.7071,0.7071),(0.7071$, $-0.7071),(0.7071,0.7071)$; the 4 star points $(1,0),(-1,0),(0$, $1),(0,-1)$, one centre point $(0,0)$; and it has critI $=$ $1 / 127.9902=0.007813$ and critIII $=1.257031$. This CCD1 is rotatable.

For CCD2 with star points -1 and +1 we need $n=16$ points, the 4 cube points $(-0.7071,-0.7071),(-0.7071$, $0.7071),(0.7071,-0.7071),(0.7071,0.7071)$; the 4 star points $(-1,0),(1,0),(0,-1),(0,1)$ and one centre point $(0,0)$ replicated 8 times, and this is a rotatable and orthogonal design. This CCD2 has critI $=1 / 1023.921=0.000977$ and critIII $=1.999923$.

For $k=3$ factors the Doehlert design has $n=13$ and crit $I=$ $1 / 254.3726716=0.003931$ and critIII $=0.487394$. This Doehlert design is nearly rotatable.

The Box-Behnken design combines $2^{2}$ factorial designs of 2 factors with the zero level of a third factor and a centre point $(0,0,0)$. The $n=13$ experimental units are $(1,1,0),(1$, $-1,0),(-1,1,0),(-1,-1,0),(1,0,1),(1,0,-1),(-1,0,1),(-1,0$, $-1),(0,1,1),(0,1,-1),(0,-1,1),(0,-1,-1),(0,0,0)$ and has critI $=1 / 8388608=1.19209 \mathrm{E}-07$ and critIII $=15.62979$. This Box-Behnken design is not-rotatable.

The CCD1 with $n=15$ experimental units has the 8 cube points: $(-0.7071,-0.7071,-0.7071),(0.7071,-0.7071$, $-0.7071),(-0.7071,0.7071,-0.7071),(0.7071,0.7071$, $-0.7071),(-0.7071,-0.7071,0.7071),(0.7071,-0.7071$, 0.7071), (-0.7071, 0.7071, 0.7071), (0.7071, 0.7071, 0.7071); the 6 star points $(-1,0,0), 1,0,0),(0,-1,0),(0,1,0),(0,0$, $-1),(0,0,1)$ and the centre point $(0,0,0)$. This CCD1 is rotatable and non-orthogonal and has critI $=1 / 82926.5=$ $1.205887 \mathrm{E}-05$ and critIII $=2.907188$.

The CCD2 with $n=32$ experimental units has the 8 cube points: $(-0.7071,-0.7071,-0.7071),(0.7071,-0.7071$, $-0.7071), \quad(-0.7071,0.7071,-0.7071),(0.7071,0.7071$, $-0.7071),(-0.7071,-0.7071,0.7071),(0.7071,-0.7071$, 0.7071), (-0.7071, 0.7071, 0.7071), (0.7071, 0.7071, 0.7071); the 6 star points which occur twice: $(-1,0,0), 1,0,0),(0,-1$, $0),(0,1,0),(0,0,-1),(0,0,1)$ and the centre points which occur 12 times $(0,0,0)$. This CCD2 is rotatable and orthogonal and has crit $I=1 / 8387160.073=1.1923 \mathrm{E}-07$ and critIII $=6.349239$.

\section{REFERENCES}

[1] Box, G.E.P. and Behnken, D.W. (1960): Some new three-level designs for the study of quantitative variables, Technometrics, $2,455-475$.

[2] Box, G.E.P. and Wilson, K.B. (1951): On the experimental attainment of optimum conditions, Journal of the Royal Statistical Society, Ser. B, 13, $1-45$.

[3] Draper, N.T. and Lin, D.K.T. (1990): Small response-surface designs, Technometrics 32, $187-194$.

[4] Doehlert, D.H. (1970): Uniform shell designs, Journal of the 
Royal Statistical Society, Ser. C, 19, 231 - 239.

[5] Doehlert, D.H. and Klee, V.L. (1972): Experimental designs through level reduction of the d-dimensional cuboctahedron, Discrete Mathematics, 2, 309 - 334.
[6] Rasch, D., Pilz, J., Verdooren, R. and Gebhardt, A. (2011): Optimal experimental design with $R$, Chapman \& Hall /CRC, Boca Raton FL, USA. 